\title{
Marginal pulmonary function should not preclude lobectomy in selected patients with non-small cell lung cancer
}

\author{
Matthew D. Taylor, MD, ${ }^{\mathrm{a}}$ Damien J. LaPar, MSc, MD, ${ }^{\mathrm{a}}$ James M. Isbell, MD, MSCI, ${ }^{\mathrm{a}}$ \\ Benjamin D. Kozower, MD, MPH, ${ }^{\mathrm{a}}$ Christine L. Lau, MD, MBA, ${ }^{\mathrm{a}}$ and David R. Jones, MD ${ }^{\mathrm{a}, \mathrm{b}}$
}

\begin{abstract}
Objective: Current clinical trials are investigating the role of stereotactic body radiation therapy (SBRT) versus sublobar resection for patients with non-small cell lung carcinoma (NSCLC) and marginal pulmonary function tests (M-PFTs). We compared the outcomes of patients undergoing lobectomy with M-PFTs characterized by 2 accepted M-PFT criteria.
\end{abstract}

Methods: A total of 1,259 consecutive patients underwent lobectomy for NSCLC between 1999 and 2011. Patients were stratified into 2 classifications of M-PFT: American College of Surgeons Oncology Group (ACOSOG) Z4099/Radiation Therapy Oncology Group (RTOG) 1021 trial or American College of Chest Physicians (ACCP) criteria. There were 206 patients classified as having M-PFT according to ACOSOG Z4099/RTOG 1021 criteria and 131 patients classified as having M-PFT by ACCP criteria. The primary endpoints of the study were post-operative complications and survival.

Results: Median follow-up was 3.8 years. Cox-proportional survival analysis found that pathologic stage $(P<.001)$, age $(P<.001)$, and higher Zubrod functional status $(P<.001)$ were independent predictors of mortality. Using multivariable analysis for major morbidity, M-PFT status was not associated with the development of a major complication following lobectomy $(P=.68)$. M-PFT classification was not an independent predictor of mortality when controlling for other variables (ACOSOG Z4099/RTOG 1021 [ $P=.34$ ]; ACCP criteria $[P=.83])$. A composite major morbidity analysis for major morbidity following lobectomy showed no association between clinicopathologic variables or M-PFTs and the occurrence of a major postoperative morbidity.

Conclusions: In carefully selected patients with M-PFTs, lobectomy for NSCLC can be performed with acceptable morbidity and mortality. These results need to be considered when deciding if a patient should undergo lobectomy or other therapies for resectable NSCLC. (J Thorac Cardiovasc Surg 2014;147:738-46)

Recent prospective clinical trials have investigated the use of stereotactic body radiotherapy (SBRT) (Radiation Therapy Oncology Group [RTOG] 0236), sublobar resection (American College of Surgeons Oncology Group [ACOSOG] Z4032), and radiofrequency ablation (RFA) (ACOSOG Z4033) for early stage non-small cell lung carcinoma (NSCLC) for patients who have been considered medically inoperable or at high-risk for surgical intervention. ${ }^{1}$ Although results for ACOSOG Z4032 and ACOSOG Z4033 have not been published to date, the phase II trial of SBRT (ie, RTOG 0236) demonstrated a 3-year overall survival of $56 \%$ and disease-free survival of $48 \%$ for patients with stage I NSCLC. ${ }^{2}$ The results of this study

From the Division of Thoracic and Cardiovascular Surgery, ${ }^{a}$ Department of Surgery, University of Virginia, Charlottesville, Va; and Department of Surgery, ${ }^{\mathrm{b}}$ Memorial Sloan-Kettering Cancer Center, New York, NY.

Disclosures: Authors have nothing to disclose with regard to commercial support.

Read at the 93rd Annual Meeting of The American Association for Thoracic Surgery, Minneapolis, Minnesota, May 4-8, 2013.

Received for publication May 8, 2013; revisions received Aug 11, 2013; accepted for publication Sept 30, 2013; available ahead of print Nov 18, 2013.

Address for reprints: David R. Jones, MD, Department of Surgery, Memorial Sloan-Kettering Cancer Center, 1275 York Ave, Box 7, New York, NY 10065 (E-mail: jonesd2@mskcc.org).

$0022-5223 / \$ 36.00$

Copyright (c) 2014 by The American Association for Thoracic Surgery

http://dx.doi.org/10.1016/j.jtcvs.2013.09.064 resulted in the randomized clinical trial (ie, ACOSOG Z4099/RTOG 1021) comparing SBRT to sublobar resection in high-risk patients with stage I disease based on marginal pulmonary function tests (M-PFTs) and comorbidities.

Although interest in SBRT and sublobar resection for early stage NSCLC in high-risk patients has grown over the past 10 years, the current gold standard therapy for NSCLC is lobectomy. Ginsberg and colleagues ${ }^{3}$ previously demonstrated a 3-fold increase in locoregional recurrences in patients with T1N0 disease undergoing wedge resection or segmentectomy. In a retrospective analysis, ${ }^{4}$ we showed that sublobar resection is associated with a 4-fold increase in locoregional recurrence after R0 resection. Additionally, some patients with M-PFTs have centrally located tumors or larger tumors and lobectomy is often required to obtain an R0 resection.

We investigated the outcomes of patients undergoing lobectomy for NSCLC classified as high risk for surgery using both the inclusion criteria for the ACOSOG Z4099/ RTOG 1021 trial and American College of Chest Physicians (ACCP) criteria.

\section{METHODS}

\section{Patient Selection}

A retrospective analysis of a prospectively maintained General Thoracic Surgery Database at the University of Virginia from July 1999 through 


\section{Abbreviations and Acronyms \\ ACCP = American College of Chest Physicians \\ $\mathrm{ACSOG}=$ American College of Surgeons Oncology Group \\ $\mathrm{CT}=$ computed tomography \\ DLCO = diffusing capacity for carbon monoxide \\ FEV1 = forced expiratory volume in 1 second \\ $\mathrm{M}$-PFT $=$ marginal pulmonary function test \\ NSCLC $=$ non-small cell lung carcinoma \\ RFA = radiofrequency ablation \\ RTOG = Radiation Therapy Oncology Group \\ SBRT = stereotactic body radiotherapy \\ STS $=$ The Society of Thoracic Surgeons}

August 2011 was performed. The General Thoracic Surgery Database is maintained by the Division of Thoracic and Cardiovascular Surgery and includes all data fields contained within the Society of Thoracic Surgeons (STS) General Thoracic Surgery Database in addition to other important clinicopathologic variables. All standardized variables analyzed represent STS definitions. Clinicopathologic variables were selected a priori and included age, sex, pathologic stage, Zubrod function status, hypertension, coronary artery disease, peripheral vascular disease, diabetes (requiring hypoglycemic medications), M-PFT status, and need for home oxygen therapy (continuous or nocturnal). The Human Investigations Committee at the University of Virginia approved this study.

A study population of 1259 patients who underwent a lobectomy for NSCLC was selected for analysis. Patients with carcinoid histology, second lung primary lesions, and sublobar resection were excluded from the study. The majority of patients had clinical stage I NSCLC. Pathology reports were staged using the seventh edition of the American Joint Cancer Committee/Union Internationale Contre le Cancer criteria. Postoperatively, patients were initially seen at 2 to 3 weeks by the thoracic surgeon, and then again every 6 months following resection with contrast-enhanced chest computed tomography (CT) for 5 years. Subsequent to this visit, either a chest roentgenogram or CT of the chest was reviewed annually. Positron emission tomography with CT or magnetic resonance imaging was used as clinically warranted. All visits were completed in concert with the referring oncologist when indicated. The primary outcome of interest was to examine postoperative complications and survival in patients with M-PFTs compared with patients with non-M-PFTs following lobectomy for NSCLC.

\section{Classification and Calculation of M-PFT}

All patients had pulmonary spirometry performed with measurement of forced vital capacity, forced expiratory volume in 1 second (FEV1), and diffusing capacity for carbon monoxide (DLCO). Patients were stratified into two M-PFT categories: ACOSOG Z4099/RTOG 1021 randomized trial for SBRT versus sublobar resection (FEV1 $\leq 50 \%$ or DLCO $\leq 50 \%$ or age $>75$ years and FEV1 $50 \%-60 \%$ or age $>75$ years and DLCO $50 \%-60 \%$ ) and ACCP criteria (postoperative FEV1 $<40 \%$ or postoperative DLCO $<40 \%$ ). Although the majority of patients had pre-resection quantitative perfusion scans, not all patients did. Therefore, for the purposes of this study the post-resectional corrected postoperative FEV1 and postoperative DLCO were calculated based on the remaining bronchopulmonary segments following lobectomy.

\section{Composite Major Morbidity Model}

To compare the likelihood of a major postoperative morbidity in patients undergoing lobectomy for NSCLC, we created a composite major morbidity model that would define associations between clinicopathologic variables and the postoperative occurrence of at least 1 major morbidity. We performed 2 separate statistical analyses investigating the composite major morbidity model using the entire cohort based upon separate M-PFT definitions. The first analysis investigated the composite major morbidity model of patients using the ACOSOG Z4099/RTOG 1021 trial definition included 206 patients with M-PFT and 1053 patients with non-M-PFTs. The second analysis of composite major morbidity used the ACCP M-PFT definition and included 131 patients with M-PFT and 1128 patient with non-M-PFTs. The complications included in the model were postoperative pneumonia, myocardial infarction, reintubation, renal failure, and atrial arrhythmia. The risk-adjusted composite major morbidity and the association with clinicopathologic variables were assessed using Cox-proportional hazards regression analysis.

\section{Statistical Analysis}

The effect of PFT status on patient short-term and long-term outcomes was assessed using standard hypothesis testing statistical analyses. Categorical variables are presented as a percentage of the group of origin, whereas continuous variables are presented as mean \pm standard deviation (for normally distributed data) or median (interquartile range) (for non-normally distributed data). Descriptive and unadjusted differences between marginal and non-marginal study cohorts were determined using the $\chi^{2}$ or Fisher exact test for categorical variable comparisons, whereas single-factor analysis of variance or the Mann Whitney $U$ test were used to compare continuous data where appropriate.

The effect of PFT status on overall survival was assessed using unadjusted and risk-adjusted survival analyses. Two separate statistical analyses investigating overall survival were performed for all patients included in the study based upon separate M-PFT definitions. The first analysis investigated the overall survival of patients using the ACOSOG Z4099/RTOG 1021 trial definition, which included 206 patients with M-PFTs and 1053 patients with non-M-PFTs. The second analysis of overall survival used the ACCP M-PFT definition and included 131 patients with M-PFTs and 1128 patient with non-M-PFTs. The unadjusted relationship between PFT status and survival was assessed using Kaplan-Meier analysis with the log-rank test, whereas risk-adjusted survival differences as a function of PFT status were assed using Cox-proportional hazards regression analysis.

Calculated test statistics were used to determine all probability estimates, and standard statistical significance was set to $\alpha<0.05$. All statistical analyses were performed using PASW version 19.0.0 (IBM Corp, Armonk, NY).

\section{RESULTS}

The demographic characteristics of the populations investigated are shown in Table 1. Using ACOSOG Z4099/RTOG 1021 M-PFT criteria, 206 patients were classified as having M-PFTs. With ACCP M-PFT criteria, 131 patients were classified as having M-PFTs. The mean age of patients and sex distribution with M-PFTs were not significantly different compared with the non-marginal group in both categories. Of all patients within the study, $6.1 \%$ had induction therapy before resection. Both M-PFT categories had equal proportions of both upper and lower lobectomies. Middle lobectomies were more common in both categories of M-PFTs compared with patients with non-M-PFTs. There was no significant difference in the proportion of pathologic stages between both categories of M-PFT compared with patients with non-M-PFTs. The proportion of M-PFT patients with a 
TABLE 1. Patient demographics

\begin{tabular}{|c|c|c|c|c|c|c|}
\hline \multirow[b]{2}{*}{ Variable } & \multicolumn{3}{|c|}{ ACOSOG Z4099/RTOG 1021 M-PFT criteria $(n=1259)$} & \multicolumn{3}{|c|}{ ACCP M-PFT criteria $(n=1259)$} \\
\hline & $\begin{array}{l}\text { M-PFTs } \\
(\mathrm{n}=206)\end{array}$ & $\begin{array}{l}\text { Non-M-PFTs } \\
(\mathbf{n}=\mathbf{1 0 5 3})\end{array}$ & $\boldsymbol{P}$ & $\begin{array}{c}\text { M-PFTs } \\
(\mathbf{n}=131)\end{array}$ & $\begin{array}{c}\text { Non-M-PFTs } \\
(\mathbf{n}=\mathbf{1 1 2 8})\end{array}$ & $\boldsymbol{P}$ \\
\hline Age, y (mean \pm standard deviation) & $69.4 \pm 9.6$ & $66.8 \pm 10.5$ & .34 & $66.4 \pm 9.7$ & $66.0 \pm 10.1$ & .48 \\
\hline Sex (men:women) & 104:102 & $507: 546$ & .57 & $65: 68$ & $538: 588$ & .92 \\
\hline FEV1, \% (median [range]) & $64(15-136)$ & $84(51-169)$ & .001 & $57(15-120)$ & $77(45-169)$ & .001 \\
\hline DLCO, \% (median [range]) & $49(13-141)$ & $79(51-156)$ & .001 & $48(13-102)$ & $81(48-156)$ & .001 \\
\hline FVC, \% (median [range]) & $77(22-140)$ & $89(52-151)$ & .001 & $69(22-137)$ & $89(43-151)$ & .001 \\
\hline Median length of stay, d & 6.2 & 5.4 & .78 & 6.4 & 5.1 & .67 \\
\hline \multicolumn{7}{|l|}{ Tumor location } \\
\hline Upper lobe & $138(66.9)$ & $698(66.2)$ & .95 & $93(70.7)$ & $743(65.9)$ & .20 \\
\hline Middle lobe & $16(7.9)$ & $41(3.9)$ & .04 & $14(10.5)$ & $43(3.8)$ & .001 \\
\hline Lower lobe & $52(25.2)$ & $314(29.9)$ & .23 & $26(19.8)$ & $340(30.3)$ & .17 \\
\hline \multicolumn{7}{|l|}{ Pathologic stage } \\
\hline I & $141(66.5)$ & $702(64.9)$ & .69 & $84(64.4)$ & 759 (66.9) & 49 \\
\hline II & $46(21.6)$ & $217(20.0)$ & .58 & $30(22.8)$ & $233(20.3)$ & .57 \\
\hline III & $16(10.5)$ & $118(13.6)$ & .22 & $15(11.5)$ & $119(11.0)$ & .76 \\
\hline IV & $3(1.4)$ & $16(1.5)$ & 1.0 & $3(2.3)$ & $16(1.8)$ & .43 \\
\hline \multicolumn{7}{|l|}{ Comorbidities } \\
\hline Hypertension & $56(27.3)$ & $246(23.4)$ & .25 & $34(26.3)$ & $268(23.7)$ & .59 \\
\hline Coronary artery disease & $39(19.3)$ & $210(20.0)$ & .74 & $27(20.3)$ & $222(19.6)$ & .82 \\
\hline Peripheral vascular disease & $62(5.9)$ & $99(9.4)$ & .65 & $10(7.5)$ & $151(13.4)$ & .07 \\
\hline Diabetes & $21(10.1)$ & $140(13.3)$ & .20 & $12(9.5)$ & $149(13.2)$ & .21 \\
\hline Zubrod functional status $>1$ & $27(13.1)$ & $69(6.5)$ & .001 & $15(11.3)$ & $81(7.2)$ & .08 \\
\hline Home oxygen therapy & $22(10.7)$ & $37(3.5)$ & .001 & $12(9.5)$ & $47(4.2)$ & .01 \\
\hline \multicolumn{7}{|l|}{ Postoperative complications } \\
\hline 30-d mortality, $\%$ & 0.5 & 1.4 & .10 & 0.8 & 1.3 & .09 \\
\hline Pneumonia, \% & 10.4 & 5.6 & .002 & 10.6 & 5.8 & .01 \\
\hline Re-intubation, \% & 5.3 & 1.1 & .62 & 4.6 & 3.7 & .44 \\
\hline Myocardial infarction, \% & 2.7 & 0.7 & .008 & 2.3 & 0.8 & .01 \\
\hline Acute renal failure, $\%$ & 2.8 & 0.7 & .01 & 2.3 & 0.8 & .01 \\
\hline Supraventricular arrhythmia, \% & 12.4 & 9.3 & .16 & 13.7 & 9.4 & .06 \\
\hline
\end{tabular}

Values are presented as n (\%) unless otherwise noted. ACOSOG, American College of Surgeons of Oncology; RTOG, Radiation Therapy Oncology Group; M-PFT, marginal pulmonary function test; $A C C P$, American College of Chest Physicians; FEVI, forced expiratory volume at 1 second; $D L C O$, carbon monoxide diffusing capacity; FVC, forced vital capacity.

Zubrod functional status $>1$ was significantly greater than patients with non-M-PFTs $(13.1 \%$ vs $6.5 \% ; P=.001)$. Additionally, a larger proportion of patients with M-PFT required home oxygen before surgery $(10.3 \%$ vs $3.5 \%)$. There was no significant difference in 30-day mortality in both M-PFT classifications when compared with non-M-PFT patients. The M-PFT group had a higher 90-day mortality compared with non-M-PFT patients $(3.8 \%$ vs $2.6 \%)$ but did not reach statistical significance $(P=.15)$. The incidence of preoperative renal dysfunction was not significantly different between patients with M-PFTs and those patients with non-M-PFTs (ACOSOG Z4099/RTOG 1021 criteria $P=.67$, ACCP criteria $P=.73$ ). Both M-PFT categories were associated with a higher incidence of postoperative pneumonia, myocardial infarction, and acute renal failure compared with non-M-PFT patients.

Table 2 illustrates the multivariable analysis comparing overall survival between patients with M-PFTs and
non-M-PFTs. In both M-PFT categories, pathologic stage, age, and Zubrod status were all associated with decreased overall survival. Pathologic stage was the variable with the strongest association to overall survival (Wald test, 35.70 and 36.42) whereas M-PFT status was not associated with overall survival. Preoperative radiation therapy was associated with a significant decrease in overall survival $(P=.04)$. We found there was no significant difference in overall survival when comparing patients who underwent upper lobectomy to those who underwent lower lobectomy (4.3 vs 4.9 years, respectively; $P=.93$ ). Figure 1 illustrates the risk-adjusted overall survival curves for both categories of M-PFT classifications. In both M-PFT categories, M-PFTs were not associated with a decrease in overall survival compared with patients with non-marginal PFTs.

We then created a composite major morbidity model (Table 3) that included postoperative pneumonia, myocardial infarction, reintubation, acute renal failure, and supraventricular tachycardia to determine what 
TABLE 2. Multivariable analysis for overall survival

\begin{tabular}{|c|c|c|c|c|c|c|}
\hline \multirow[b]{2}{*}{ Variable } & \multicolumn{3}{|c|}{ ACOSOG Z4099/RTOG 1021 M-PFT criteria $(n=1259)$} & \multicolumn{3}{|c|}{ ACCP M-PFT criteria $(n=1259)$} \\
\hline & Wald & $\boldsymbol{P}$ & HR & Wald & $\boldsymbol{P}$ & HR \\
\hline Pathologic stage* & 35.70 & $<.001$ & & 36.42 & $<.001$ & \\
\hline IB & 2.52 & .11 & 1.20 & 2.56 & .11 & 1.20 \\
\hline IIA & 0.194 & .66 & 0.92 & 0.42 & .52 & 0.88 \\
\hline IIB & 2.06 & .15 & 1.25 & 2.08 & .15 & 1.25 \\
\hline IIIA & 14.80 & $<.001$ & 1.81 & 14.00 & $<.001$ & 1.79 \\
\hline IIIB & 19.08 & $<.001$ & 2.34 & 19.70 & $<.001$ & 2.38 \\
\hline IV & 6.88 & .009 & 2.38 & 7.05 & .008 & 2.42 \\
\hline Age & 34.64 & $<.001$ & 1.03 & 33.47 & $<.001$ & 1.03 \\
\hline Zubrod status $\dagger$ & 23.60 & $<.001$ & & 24.50 & $<.001$ & \\
\hline 1 & 10.23 & .001 & 1.37 & 12.29 & $<.001$ & 1.41 \\
\hline 2 & 16.55 & $<.001$ & 2.02 & 15.87 & $<.001$ & 2.01 \\
\hline 3 & 4.93 & .03 & 2.38 & 4.96 & .03 & 2.38 \\
\hline Hypertension & 3.86 & .05 & 0.77 & 3.14 & .08 & 0.79 \\
\hline Coronary artery disease & 2.27 & .13 & 1.20 & 2.29 & .13 & 1.20 \\
\hline Peripheral vascular disease & 1.42 & .23 & 1.28 & 1.13 & .29 & 1.24 \\
\hline Sex (female) & 1.32 & .25 & 0.90 & 1.67 & .20 & 0.89 \\
\hline Diabetes & 1.26 & .26 & 1.17 & 0.71 & .40 & 1.13 \\
\hline M-PFT status & 0.91 & .34 & 1.12 & 0.48 & .83 & 0.97 \\
\hline Home oxygen therapy & 0.47 & .49 & 0.84 & 0.53 & .46 & 0.83 \\
\hline
\end{tabular}

ACOSOG, American College of Surgeons of Oncology; RTOG, Radiation Therapy Oncology Group; M-PFT, marginal pulmonary function test; ACCP, American College of Chest Physicians; $H R$, hazard ratio. *Reference stage: $1 \mathrm{~A}$. †Reference: 0.

clinicopathologic variables may be associated with the occurrence of at least 1 major complication following lobectomy. There was no significant difference in the incidence of major postoperative morbidity on univariate

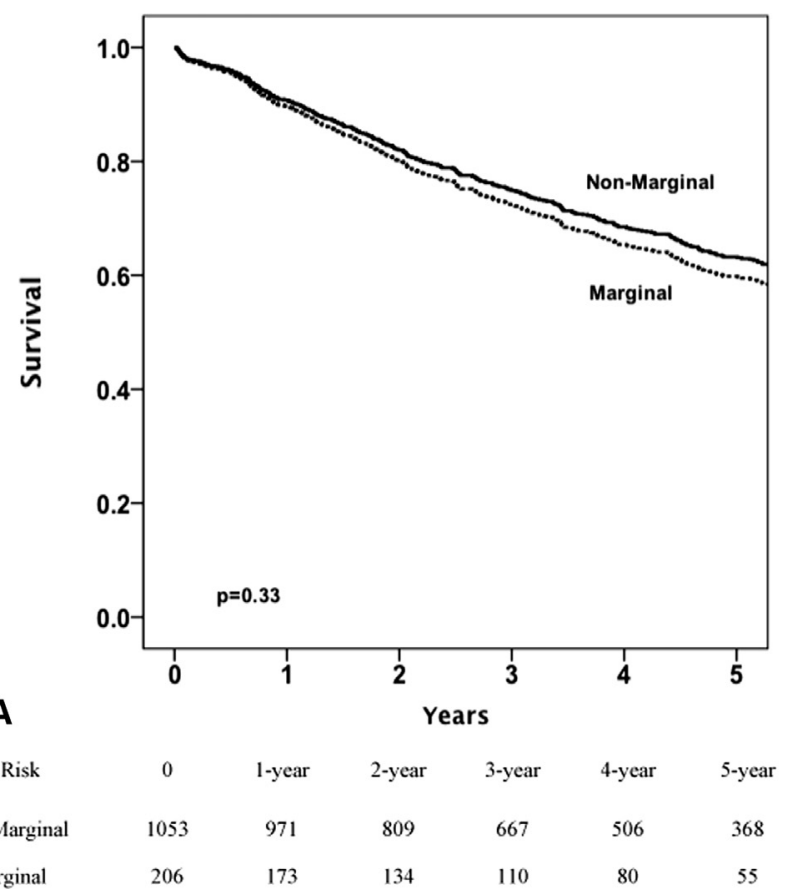

analysis when comparing patients who underwent upper lobectomy to lower lobectomy $(P=.52)$. A multivariable analysis was conducted using this morbidity model. The only variable associated with a major complication was

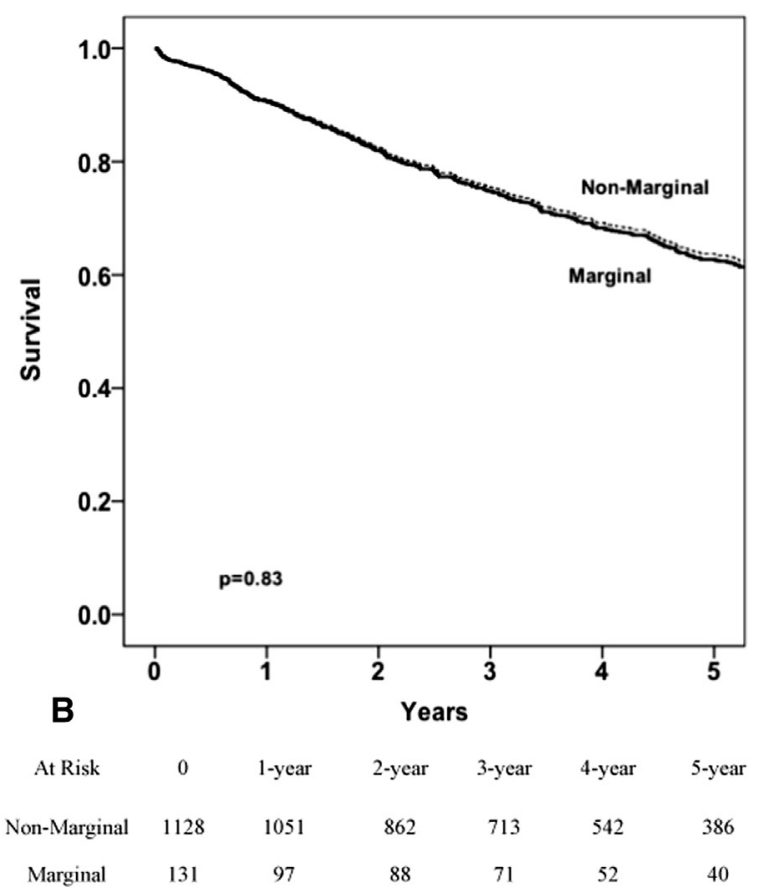

FIGURE 1. A, Risk-adjusted overall survival curve for American College of Surgeons Oncology Group Z4099/Radiation Therapy Oncology Group 1021 trial or American College of Chest Physicians (ACCP) marginal-pulmonary function test (M-PFIT) criteria. B, Risk-adjusted overall survival curve for ACCP M-PFT criteria. 
TABLE 3. Multivariable analysis for composite morbidity model

\begin{tabular}{|c|c|c|c|c|c|c|}
\hline \multirow[b]{2}{*}{ Variable } & \multicolumn{3}{|c|}{ ACOSOG Z4099/RTOG 1021 M-PFT criteria $(n=1259)$} & \multicolumn{3}{|c|}{ ACCP M-PFT criteria $(n=1259)$} \\
\hline & Wald & $\boldsymbol{P}$ & HR & Wald & $\boldsymbol{P}$ & HR \\
\hline Pathologic stage* & 3.61 & .73 & & 4.72 & .58 & \\
\hline IB & 0.44 & .51 & 1.14 & 0.05 & .83 & 1.04 \\
\hline IIA & 0.37 & .54 & 0.83 & 1.09 & .30 & 0.72 \\
\hline IIB & 0.009 & .92 & 1.03 & 0.02 & .89 & 1.04 \\
\hline IIIA & 0.12 & .73 & 1.10 & 0.23 & .63 & 1.14 \\
\hline IIIB & 0.02 & .88 & 0.96 & 0.03 & .86 & 0.95 \\
\hline IV & 2.40 & .12 & 2.54 & 2.97 & .08 & 2.86 \\
\hline Age & 5.10 & .02 & 1.02 & 2.50 & .11 & 1.01 \\
\hline Zubrod status $\dagger$ & 5.10 & .16 & & 5.09 & .17 & \\
\hline 1 & 2.67 & .10 & 0.76 & 3.44 & .06 & 0.73 \\
\hline 2 & 0.52 & .47 & 1.19 & 0.18 & .67 & 1.11 \\
\hline 3 & 0.21 & .65 & 1.27 & 0.10 & .76 & 1.18 \\
\hline Hypertension & 0.51 & .48 & 1.14 & 0.17 & .68 & 1.08 \\
\hline Coronary artery disease & 0.19 & .66 & 1.09 & 0.19 & .66 & 1.09 \\
\hline Peripheral vascular disease & 2.20 & .14 & 0.66 & 0.72 & .40 & 0.79 \\
\hline Sex (female) & 0.01 & .91 & 0.98 & 0.10 & .76 & 0.95 \\
\hline Diabetes & 1.14 & .29 & 0.78 & 1.46 & .23 & 0.75 \\
\hline M-PFT status & 0.17 & .68 & 0.93 & 2.23 & .14 & 0.65 \\
\hline Home oxygen therapy & 1.00 & .32 & 1.31 & 3.42 & .06 & 1.65 \\
\hline
\end{tabular}

ACOSOG, American College of Surgeons of Oncology; RTOG, Radiation Therapy Oncology Group; $M-P F T$, marginal pulmonary function test; ACCP, American College of Chest Physicians; HR, hazard ratio. *Reference stage: $1 \mathrm{~A}$. $\dagger$ Reference: 0.

increasing age within the ACOSOG Z4099/RTOG 1021 criteria for M-PFTs. Neither M-PFT classification was associated with an increased risk of developing any of the 5 complications included in the composite model. Additionally, we found that current smoking status and induction therapy were not associated with an increased risk of a major complication postoperatively $(P=.65$ and .81 , respectively).

\section{DISCUSSION}

This study demonstrates that M-PFTs, as classified by 2 methodologies, are not associated with a decrease in overall survival in patients undergoing lobectomy for NSCLC. Also, when risk-adjusting for preoperative and pathologic variables, the incidence of significant morbidity was not greater in selected patients with M-PFTs who underwent lobectomy.

Currently, the standard resectional strategy for patients with NSCLC who have adequate pulmonary reserve and are deemed low to moderate risk for surgery is lobectomy. For patients considered medically inoperable or at high risk for surgery, sublobar resection, brachytherapy/wedge resection, SBRT, and RFA have all been proposed as alternative treatment strategies. Previous studies have demonstrated an increased incidence of locoregional recurrence following sublobar resection compared with lobectomy. ${ }^{3-6}$ Comparison of oncologic outcomes between sublobar resection and lobectomy will be elucidated with the results of the Cancer and Leukemia Group B 140503 trial.
Robinson and colleagues ${ }^{7}$ compared the outcomes of patients with stage I NSCLC undergoing lobectomy or pneumonectomy versus SBRT in 338 patients. Patients who underwent SBRT in this study had median FEV1 of $51 \%$ compared with patients who underwent lobar resection with a median FEV1 of $79 \%(P<.0001)$. DLCO was significant lower in the SBRT group versus lobar resection group $(62 \%$ vs $79 \% ; P=.001)$. In this comparison, 4-year local control was $99 \%$ for the surgical arm versus $94 \%$ in the SBRT arm, which was not significantly different. Overall survival favored lobar resection ( $64 \%$ vs $30 \%)$ but disease-specific survival was not significant between groups. The difficulty with this analysis is that the 2 populations studied are inherently different based on pulmonary function, comorbid conditions, and tumor size.

Results from RFA studies for stage I NSCLC have demonstrated an overall 2-year survival rate between $68 \%$ and $72 \%{ }^{8,9}$ However, these studies demonstrated significant locoregional failure rates associated with RFA. Pennathur and colleagues ${ }^{8}$ showed $42 \%$ locoregional failure at 27 months from RFA treatment in patients with a median FEV1 of $29 \%$. In addition, Lanuti and colleagues ${ }^{9}$ showed an $80 \%$ locoregional failure rate following RFA for tumors $>3 \mathrm{~cm}$ in size in patients with a mean FEV1 of $62 \%$.

Use of current marginal PFT criteria as a sole means to exclude patients from definitive resection and for inclusion in studies for alternative therapies such as SBRT, RFA, or sublobar resection are not supported by our findings. 
The results of our study with the 2 most commonly used M-PFT classifications suggests that the criteria for a high-risk surgical candidate within SBRT trials may not be strict enough and a significant proportion of patients undergoing SBRT could in fact tolerate a lobectomy.

We chose to limit our study to patients with M-PFTs who underwent lobectomy. Previous studies have investigated the predictive ability of pulmonary function tests on outcomes following lobectomy. Berry and colleagues ${ }^{10}$ evaluated the predictors of pulmonary complications in 340 patients with a FEV1 or DLCO of $60 \%$ or less who underwent thoracoscopy $(\mathrm{n}=173)$ or thoracotomy $(\mathrm{n}=164)$ and subsequent lobectomy. DLCO and FEV1 were found to be predictors of pulmonary complications in patients undergoing thoracotomy but not thoracoscopy. Both M-PFT criteria used in our study were stricter than that study. Several studies show the incidence of pulmonary complications and mortality are acceptable in patients undergoing lobectomy with postoperative pulmonary function $<50 \%$ predicted. ${ }^{11,12}$ Puente-Maestu and colleagues $^{13}$ developed an algorithm using postoperative predicted peak oxygen uptake $>10 \mathrm{~mL} / \mathrm{kg} /$ minute to assess suitability for surgery in patients with postoperative FEV1 and/or postoperative DLCO $<40 \%$. When comparing patients with postoperative FEV1 and postoperative DLCO $>40 \%$ to patients with postoperative FEV1 and/or postoperative DLCO $<40 \%$, overall survival at 2 years was not significantly different between groups $(71 \%$ vs $57 \% ; P=.15)$. Sixty-day mortality was $6.5 \%$ in patients undergoing lobectomy and 2-year overall survival was $66 \%$.

Previous studies have investigated the association of preoperative variables on outcomes following lung cancer resection. Kozower and colleagues ${ }^{14}$ used the STS General Thoracic Surgery Database to define predictors of morbidity and mortality following lung cancer resection. Several variables were found to be predictors of mortality, including increasing Zubrod score, increasing age, and decreasing FEV1. Our study also found that increasing age and increasing Zubrod functional status was associated with an increase in mortality following lung cancer resection. Several differences exist between this study and the study published by our group previously. First, this study included only patients undergoing lobectomy, whereas the previous study also included patients undergoing pneumonectomy and sublobar resection. Second, the STS database is not internally audited for data quality, whereas our database is audited. Third, the STS database is limited to 30-day follow-up, whereas our study had a median follow-up of 3.8 years. Wright and colleagues ${ }^{15}$ also demonstrated that increasing age, Zubrod score, and decreased FEV1 were predictors of prolonged length of stay following lobectomy for lung cancer using the STS database. Length of stay was not statistically different between patients with M-PFTs compared with patients with non-M-PFTs in our study.

Our study demonstrates that classification of patients as having M-PFTs does not predict mortality or major morbidity following lobectomy. Our 30-day perioperative mortality rates for both M-PFT groups was $<1 \%$. Although the multivariable analysis of composite morbidity showed no association with the incidence of 1 major morbidity and M-PFT status, we did find a 4-fold increase in postoperative myocardial infarction and acute renal failure in the M-PFT group. This discovery may lead to changes in practice guidelines for patients with M-PFTs. In patients with M-PFTs who do not have identifiable risk factors for coronary artery disease, preoperative cardiac stress testing may be warranted to identify patients who may benefit from preoperative coronary revascularization or medical optimization before surgery. Despite no significant difference in baseline creatinine levels between M-PFT and non-M-PFT groups, M-PFT patients had a 4-fold higher incidence of acute renal insufficiency compared with non-M-PFT patients. This finding suggests that the potential need for judicious fluid management in the postoperative period may be resulting in hypovolemia and subsequent renal insufficiency. However, further studies investigating the need for renal protective strategies are needed in M-PFT patients. Future studies should investigate the minimal post-resection pulmonary function needed to avoid postoperative respiratory failure with associated morbidity and mortality taking into account the use of minimally invasive techniques, perioperative epidural catheter use, and optimization of pulmonary physiotherapy in the postoperative period.

The limitations of this study include the retrospective nature of the analysis and the possibility for selection bias. Another limitation is that our patients did not routinely undergo baseline arterial blood gas analysis, mixed venous partial pressure of oxygen testing, or echocardiogram to assess pulmonary hypertension before surgery. Therefore, we were unable to capture additional patients who would have been considered to have M-PFTs according to the ACOSOG Z4099/RTOG 1021 trial definitions.

\section{CONCLUSIONS}

M-PFTs, as classified by 2 methodologies currently being evaluated in clinical trials, are not associated with a decrease in overall survival in patients undergoing lobectomy for NSCLC. Additionally, when controlling for preoperative and pathologic variables, the incidence of having any significant morbidity following surgery was not greater in patients with M-PFTs undergoing lobectomy. This study suggests that current SBRT and sublobar resection trials characterizing patients as nonsurgical candidates based on 2 accepted M-PFT definitions may be excluding patients from undergoing lobectomy, the current 
gold standard therapy for lung cancer. Future studies are needed to ascertain the influence of other patient factors used my experienced thoracic surgeons in determining the candidacy for lobar resection in patients with marginal pulmonary reserve.

\section{References}

1. Crabtree T, Puri V, Timmerman R, Fernando H, Bradley J, Decker PA, et al. Treatment of stage I lung cancer in high-risk and inoperable patients: comparison of prospective clinical trials using stereotactic body radiotherapy (RTOG 0236), sublobar resection (ACOSOG Z4032), and radiofrequency ablation (ACOSOG Z4033). J Thorac Cardiovasc Surg. 2013;145:692-9.

2. Timmerman R, Paulus R, Galvin J, Michalski J, Straube W, Bradley J, et al. Stereotactic body radiation therapy for inoperable early stage lung cancer. JAMA. 2010;303:1070-6.

3. Ginsberg RJ, Rubinstein LV. Randomized trial of lobectomy versus limited resection for T1 N0 non-small cell lung cancer. Lung Cancer Study Group. Ann Thorac Surg. 1995;60:615-22.

4. Taylor MD, Nagji AS, Bhamidipati CM, Theodosakis N, Kozower BD, Lau CL, et al. Tumor recurrence after complete resection for non-small cell lung cancer. Ann Thorac Surg. 2012;93:1813-20.

5. Santos R, Colonias A, Parda D, Trombetta M, Maley RH, Macherey R, et al. Comparison between sublobar resection and 125Iodine brachytherapy after sublobar resection in high-risk patients with Stage I non-small-cell lung cancer. Surgery. 2003;134:691-7.

6. Fernando HC, Santos RS, Benfield JR, Grannis FW, Keenan RJ, Luketich JD, et al. Lobar and sublobar resection with and without brachytherapy for small stage IA non-small cell lung cancer. J Thorac Cardiovasc Surg. 2005; 129:261-7.

7. Robinson CG, DeWees TA, EI Naqa IM, Creach KM, Olsen JR, Crabtree TD, et al. Patterns of failure after stereotactic body radiation therapy or lobar resection for clinical stage I non-small-cell lung cancer. J Thorac Oncol. 2013;8:192-201.

8. Pennathur A, Luketich JD, Abbas G, Chen M, Fernando HC, Gooding WE, et al. Radiofrequency ablation for the treatment of stage I non-small cell lung cancer in high-risk patients. J Thorac Cardiovasc Surg. 2007;134:857-64.

9. Lanuti M, Sharma A, Digumarthy SR, Wright CD, Donahue DM, Wain JC, et al. Radiofrequency ablation for treatment of medically inoperable stage I non-small cell lung cancer. J Thorac Cardiovasc Surg. 2009;137:160-6.

10. Berry MF, Villamizar-Ortiz NR, Tong BC, Burfeind WR Jr, Harpole DH, D'Amico TA, et al. Pulmonary function tests do not predict pulmonary complications after thoracoscopic lobectomy. Ann Thorac Surg. 2010;89: 1044-51.

11. Magdeleinat P, Seguin A, Alifano M, Boubia S, Regnard JF. Early and long-term results of lung resection for non-small-cell lung cancer in patients with severe ventilatory impairment. Eur J Cardiothorac Surg. 2005;27:1099-105.

12. Cerfolio RJ, Allen MS, Trastek VF, Deschamps C, Scanlon PD, Pairolero PC. Lung resection in patients with compromised pulmonary function. Ann Thorac Surg. 1996;62:348-51.

13. Puente-Maestú L, Villar F, González-Casurrán G, Moreno N, Martínez Y, Simón C, et al. Early and long-term validation of an algorithm assessing fitness for surgery in patients with postoperative FEV1 and diffusing capacity of the lung for carbon monoxide $<40 \%$. Chest. 2011;139:1430-8.

14. Kozower BD, Sheng S, O'Brien SM, Liptay MJ, Lau CL, Jones DR, et al. STS database risk models: predictors of mortality and major morbidity for lung cancer resection. Ann Thorac Surg. 2010;90:875-81.

15. Wright CD, Gaissert HA, Grab JD, O'Brien SM, Peterson ED, Allen MS. Predictors of prolonged length of stay after lobectomy for lung cancer: a Society of Thoracic Surgeons General Thoracic Surgery Database risk-adjustment model. Ann Thorac Surg. 2008;85:1857-65.

\section{Discussion}

Dr Yolonda L. Colson (Boston, Mass). I want to commend you on a very nice presentation. We have seen several presentations at this conference that are investigating surgical resection in marginal patients, reflecting how important this topic really is and that we face it regularly.

I have 3 questions to look at your analysis in more depth. In the non-marginal group you have almost a $5 \%$ incidence of re-intubation. That seems unusually high. Does it affect the fact that you say there wasn't much difference in complications if the higher incidence of complications was in the non-marginal group? Among the complications that you did have, did you look at length of stay, for example, and what the ramifications of those complications were?

Dr Taylor. Thank you for your questions. There was a re-intubation rate within the non-marginal pulmonary function test (PFT) group of approximately $5.3 \%$ versus $1.1 \%$ in the marginal PFT group. However, this difference was not found to be statistically different on univariate analysis. It is not clear why the non-marginal group had a $4 \%$ higher re-intubation rate. It may be that we are more conservative in extubating patients with marginal PFTs because of concern for re-intubation. We did not include length of stay analysis or specific analysis of the ramifications of postoperative complications. I think it is important to note that on our univariate analysis there was a significant difference in a number of variables, including postoperative myocardial infarction and acute renal failure. Despite the fact that we found no significant associations on multivariable analysis, this data may provide important information about patients who have marginal PFTs and the need for additional preoperative risk assessment.

Dr Colson. In patients with marginal PFTs, we usually choose stereotactic body radiotherapy or some other ablative type of therapy. They tend to think more about quality of life issues or what the complications are, and that is often the reason that these therapies will get chosen, more so necessarily than mortality. In your presentation, we don't really see afterward what the functional status is of patients who have had a lobectomy and how many of them were not receiving oxygen before and then became oxygen dependent. What is the change that happened other than just survival and a complication? What happened to them in terms of their quality of life?

Dr Taylor. That is a great question. I agree that quality of life in the marginal PFT patient population is quite important. However, we have not performed quality-of-life assessments on patients included in this study. That information would be a great follow-up study.

Dr Colson. We know if you do a lobectomy on a patient who has an upper lobe with a large bullous emphysema or a middle lobectomy it is very different than doing a lower lobectomy on somebody who has significant perfusion to that lobe, and I don't see that broken out here. Can you characterize what characteristics in these marginal patients allow safe resection? Who did well and didn't do well in terms of what their disease looked like?

Dr Taylor. Looking at the demographics of the population, there was no difference in the proportion of upper lobectomy to lower lobectomy when comparing marginal group to nonmarginal group. We did not perform a subgroup analysis to determine, for example, what factors are important for a good outcome in patients with marginal PFTs who underwent lower lobectomy. This is a 
good question that may elucidate further characteristics that may be important to consider in patients with marginal PFTS who need resection.

Dr Colson. Is it possible that what we are really looking at is that these results confirm the ability of a surgeon to look at marginal PFTs and decide when they are not as marginal as we think?

Dr Taylor. That is a great point. Pulmonary function tests are only 1 factor in considering if a patient is eligible for resection and it is clear that a thorough evaluation of the entire clinical picture is essential when considering lobectomy in this patient population.

Dr Thomas A. D'Amico (Durham, NC). I enjoyed your presentation, Dr Taylor.

What percentage of the patients underwent thoracoscopic lobectomy?

Dr Taylor. Thoracoscopic lobectomy represented $20 \%$ of the procedures performed.

Dr D'Amico. Did you not put that in your risk model, whether it was a thoracoscopic or open lobectomy?

Dr Taylor. Given the small proportion of patients undergoing video-assisted thoracoscopic lobectomy, we did not include it. We did do a subgroup analysis that is not included in the results reported here and we did not find any significant difference between video-assisted thoracoscopic surgery and the open technique with regard to morbidity and mortality in patients with marginal PFTs. Now, if that is a reflection of our sample size and the fact that we have significantly fewer patients who underwent video-assisted thoracoscopic surgical procedures than open, that may be the case.

Dr D'Amico. Do you have any idea what percentage had pulmonary hypertension?

Dr Taylor. I do not have the percentage of patients within the study who have pulmonary hypertension. I believe that is 1 of the limitations of this study. Not having pulmonary hypertension data limits some of the inclusion criteria within the clinical trial.

Dr K. Robert Shen (Rochester, Minn). I enjoyed your presentation very much and I think it is a very timely analysis.

One of the things that all thoracic surgeons do when evaluating if patients with marginal pulmonary function can tolerate surgery is to factor in other things that we inherently know will affect that patient's risk profile. Although there isn't much prospective data to prove that getting current smokers to stop smoking for a period of time before surgery and putting them through a preoperative pulmonary rehabilitation program has a positive effect in getting a patient with marginal PFT through an operation safely, I am more inclined in that patient compared with a patient with good PFT, to say, "I'm not going to offer you surgery until you stop smoking and go through pulmonary rehab." Did you put into your risk model any information about whether or not those patients in the marginal group were treated differently in terms of things like that-requiring them to stop smoking, putting them through rehab-and does the data allow you to address any of those issues? I think they are quite important and we are lacking data to show if those things may be helpful in these patients.
Dr Taylor. We did not include those particular variables within our analysis. There are data that suggest that pulmonary rehab is effective for increasing exercise capacity, but there is some question as to whether there is any increase in actual PFT improvement. I agree that evaluating the effect of smoking cessation and the use of pulmonary rehab on patients with marginal PFTs may uncover another variable that may be important in risk stratifying this patient population.

Dr Raphael Bueno (Boston, Mass). Do you have 90-day mortality data? Particularly for these kinds of patients, we ought to start reporting 90-day mortality.

Dr Taylor. In the non-marginal group, our 90-day mortality was $2.6 \%$ and in the marginal group it was $3.8 \%$.

Dr Bueno. So that is substantially up from 30-day mortality.

Dr Taylor. That is correct. It was not statistically significant on univariate analysis.

Dr Bueno. So, again, reinforcing the need to answer that question.

Dr Thomas K. Waddell (Toronto, Ontario, Canada). I enjoyed your presentation.

I am convinced by your data that the current criteria by which these trials have been constructed are not good predictors. I think that is pretty obvious. The question is if there are better predictors. I think there are 2 subsets of things you should consider. How many patients went on to stereotactic body radiation therapy and what did their profile look like by these same measures; that is, do you actually just use a lower threshold of the same measures, or are you not measuring to these criteria and not based on the right measure? I am interested in to what extent these marginal candidates had mixed venous partial pressure of oxygen measurements, forced vital capacity measurements to really determine that forced expiratory volume in 1 second below a certain level is actually an obstructive physiology, and quantitative ventilation/perfusion lung scan, so just in general, the use of ancillary measures to more precisely refine your patient selection.

Dr Taylor. Thank you Dr Waddell for these important questions. Although we did not include the profile of patients undergoing stereotactic body radiation therapy within our institution in this study, I believe your point is critical and a comparison that needs to be evaluated. With regard to our use of ancillary measures to further define pulmonary function (ie, quantitative perfusion scan and mixed venous partial pressure of oxygen), our algorithm is that if a patient has an forced expiratory volume in 1 second or carbon monoxide diffusing capacity $<50 \%$, then we proceed with a quantitative perfusion scan. If that is $<40 \%$, then we will proceed with an mixed venous partial pressure of oxygen study. If it is $>15$, we consider them eligible for resection. If the value is between 10 and 15 , it is a discussion between all parties involved, including the medical oncologists and the surgeons. Because not all patients in study had mixed venous partial pressure of oxygen or perfusion scans, we calculated predicted postoperative pulmonary function by quantifying the number of bronchopulmonary segments resected to use the same methodology on all patients.

Dr Frank A. Baciewicz (Detroit, Mich). I have 1 quick comment.

We recently studied a cohort of patients with forced expiratory volume in 1 second around $1 \mathrm{~L}$. We gave them several weeks of 
pulmonary therapy and found that you can slightly improve the forced expiratory volume in 1 second. You don't improve it significantly and it doesn't change their outcomes. This is data we are publishing in the future.

I agree that the criteria mentioned do not define high-risk patients. I was curious about patient outcomes if you subdivided them into patients who were receiving home oxygen or who had forced expiratory volume in 1 second of 1 or $<1$.

Dr Taylor. We did not perform a subgroup analysis by stratifying forced expiratory volume in 1 second of 1 or $<1$. However, the use of preoperative oxygen therapy was not found to be an independent predictor of death or major morbidity. 